\title{
Dissipation-Based Quantum Sensing of Magnons with a Superconducting Qubit
}

\author{
S. P. Wolski®, ${ }^{1,{ }^{*}}$ D. Lachance-Quirion $\odot,{ }^{1, \hbar}$ Y. Tabuchi $\odot,{ }^{1}$ S. Kono $\odot,{ }^{1,2}$ A. Noguchi $\odot,{ }^{3}$ K. Usami, ${ }^{1}$ and Y. Nakamura $\odot^{1,2, \dagger}$ \\ ${ }^{1}$ Research Center for Advanced Science and Technology (RCAST), The University of Tokyo, Meguro, Tokyo 153-8904, Japan \\ ${ }^{2}$ Center for Emergent Matter Science (CEMS), RIKEN, Wako, Saitama 351-0198, Japan \\ ${ }^{3}$ Komaba Institute for Science (KIS), The University of Tokyo, Meguro, Tokyo 153-8902, Japan
}

(Received 19 May 2020; revised 22 July 2020; accepted 29 July 2020; published 8 September 2020)

\begin{abstract}
Hybrid quantum devices expand the tools and techniques available for quantum sensing in various fields. Here, we experimentally demonstrate quantum sensing of a steady-state magnon population in a magnetostatic mode of a ferrimagnetic crystal. Dispersively coupling the magnetostatic mode to a superconducting qubit allows for the detection of magnons using Ramsey interferometry with a sensitivity on the order of $10^{-3}$ magnons $/ \sqrt{\mathrm{Hz}}$. The protocol is based on dissipation as dephasing via fluctuations in the magnetostatic mode reduces the qubit coherence proportionally to the number of magnons.
\end{abstract}

DOI: $10.1103 /$ PhysRevLett.125.117701

Quantum states are intrinsically fragile with regards to external perturbations. This property is leveraged in quantum sensing, where appropriate quantum systems can be monitored to detect a signal [1]. Superconducting qubits are attractive candidates for quantum sensors [1-8] as their large electric dipole moment enables strong coupling to electromagnetic fields $[9,10]$. Recent developments of hybrid quantum systems extend the range of applicability of qubits as quantum sensors through coupling the qubits to additional degrees of freedom [11-16].

Magnons, the quanta of collective spin excitations in magnetically ordered systems $[17,18]$, provide a rich emerging platform for advances in quantum technologies [19-26]. The presence of large quantities of magnons is typically detected using techniques such as electromagnetic induction [17], the inverse spin-Hall effect [27-29], or Brillouin light scattering [30,31]. Recently, single-shot detection of a single magnon was demonstrated in a superconducting-qubit-based hybrid quantum system, bringing the equivalent of a high-efficiency single-photon detector to the field of magnonics [16]. Such an approach, carried out by entangling the qubit and magnetostatic mode, can be used to verify that a magnon is present at a given time. However, a different measurement scheme is desired to detect a steady-state magnon population, for example when characterizing weak continuous magnon creation processes.

In this Letter, we demonstrate dissipation-based quantum sensing of magnons in a magnetostatic mode by utilizing a

Published by the American Physical Society under the terms of the Creative Commons Attribution 4.0 International license. Further distribution of this work must maintain attribution to the author(s) and the published article's title, journal citation, and DOI. transmon qubit as a quantum sensor. The hybrid device architecture allows for an engineered dispersive interaction between the qubit and magnetostatic mode, operated in the strong dispersive regime [15,16,21,32-34]. Fluctuations of the magnon number in the magnetostatic mode induce dephasing in the qubit in proportion to the magnon population and, as such, measurements of the coherence of the qubit yield information about the average number of magnons in the mode [34]. Characterization of the sensing procedure reveals a magnon detection sensitivity on the order of $10^{-3}$ magnons $/ \sqrt{\mathrm{Hz}}$, in good agreement with numerical simulations.

The hybrid system used in the experiments consists of a transmon-type superconducting qubit and a singlecrystal yttrium-iron-garnet (YIG) sphere [Fig. 1(a)], both mounted inside a three-dimensional microwave copper cavity $[19,20]$. The $\mathrm{TE}_{102}$ mode of the cavity has a dressed frequency of $8.448 \mathrm{GHz}$. The transmon qubit has a dressed frequency of $7.914 \mathrm{GHz}$ and anharmonicity of $-0.123 \mathrm{GHz}$. A magnetic circuit consisting of a pair of permanent magnets, an iron yoke, and a superconducting coil is used to apply a uniform magnetic field $\mathbf{B}_{0}$ to the YIG sphere, magnetizing it to saturation. The amplitude of the applied magnetic field, which can be tuned by changing the current in the coil, sets the frequency $\omega_{m}$ of the uniform magnetostatic mode, or Kittel mode, in the YIG sphere [35]. The Kittel mode couples to the cavity mode through a magnetic dipole interaction of $23 \mathrm{MHz}[35,36]$. Similarly, the qubit couples to the cavity mode through an electric dipole interaction of $130 \mathrm{MHz}[9,10,37,38]$. The mutual couplings with the cavity result in an effective coupling between the qubit and Kittel mode of $7.07 \mathrm{MHz}[19,20]$.

Here, the qubit and Kittel mode are detuned so that the coupling between them is dispersive, characterized by a dispersive shift per excitation, $2 \chi_{q-m}$. At such an operating point, the qubit frequency becomes dependent on the 
(a)

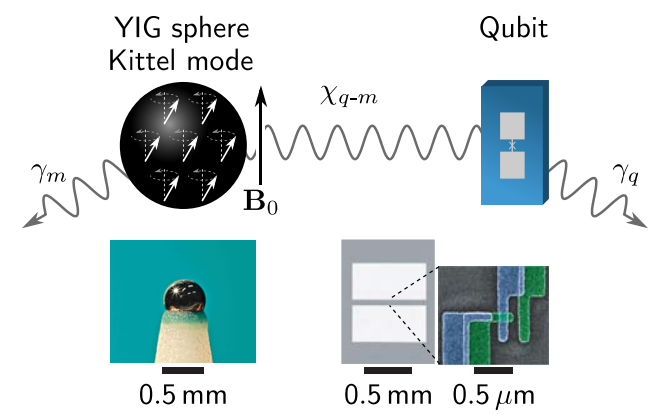

(d)

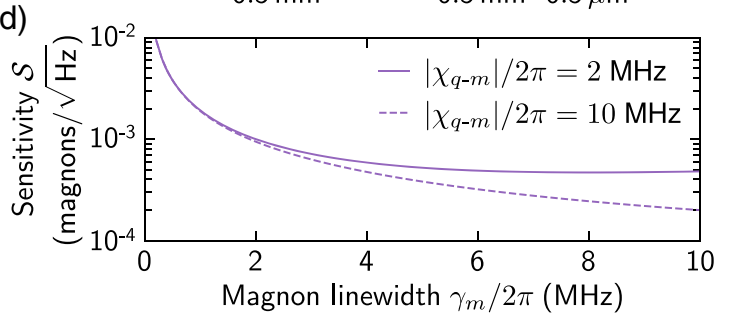

(b)

(c)
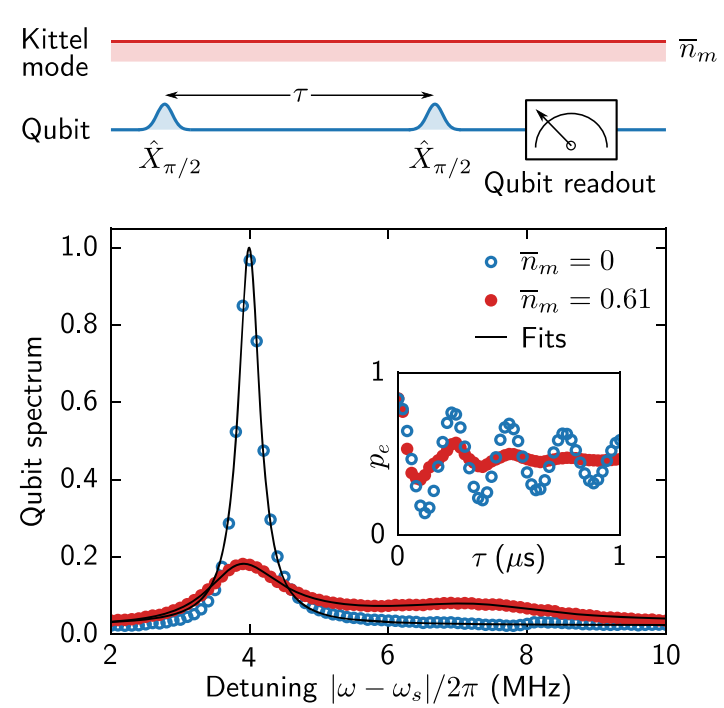

FIG. 1. (a) Photographs and schematics of a 0.5-mm-diameter single-crystal yttrium-iron-garnet (YIG) sphere and a superconducting transmon qubit. A static magnetic field $\mathbf{B}_{0}$ magnetizes the YIG sphere to saturation. The Kittel mode couples dispersively to the qubit with a dispersive shift $\chi_{q-m}$. The Kittel mode and qubit have linewidths $\gamma_{m}$ and $\gamma_{q}$, respectively. (b) Pulse sequence used for the quantum sensing of magnons. Two $\hat{X}_{\pi / 2}$ pulses with frequency $\omega_{s}$, separated by the sensing time $\tau$, are applied to the qubit as in conventional Ramsey interferometry, followed by a readout of the qubit state. A continuous drive close to resonance with the Kittel mode creates a coherent state of magnons with population $\bar{n}_{m}$. (c) Qubit spectra obtained from Ramsey interferometry with a steady-state magnon population $\bar{n}_{m}=0$ (blue circles) and 0.61 (red dots). The detuning $\left|\omega-\omega_{s}\right| / 2 \pi$ is relative to the qubit control frequency $\omega_{s} / 2 \pi$, which is itself detuned from the qubit frequency by $-4 \mathrm{MHz}$ to induce Ramsey oscillations (shown in the inset up to $\tau=1 \mu \mathrm{s}$ ) from which the spectra are obtained. Solid black lines show fits to a model. The spectra are normalized to the maximal value of the fit for $\bar{n}_{m}=0$. (d) Magnon detection sensitivity $\mathcal{S}$ calculated as a function of the Kittel mode linewidth $\gamma_{m} / 2 \pi$, for amplitudes of the qubit-magnon dispersive shift $\left|\chi_{q-m}\right| / 2 \pi=2 \mathrm{MHz}$ (solid line) and $10 \mathrm{MHz}$ (dashed line). The sensing time $\tau=0.89 \mu$ s is fixed to be equal to the qubit coherence time $T_{2}^{*}=2 / \gamma_{q}$ measured in the experiment with no magnons present.

magnon state although direct energy exchange is suppressed [32-34]. Measuring the qubit state thus yields different results depending on the number of magnons present in the Kittel mode. In the experiment, the Kittel mode frequency $\omega_{m} / 2 \pi \approx 7.781 \mathrm{GHz}$ is fixed such that the system is in the strong dispersive regime, where the dispersive shift is greater than the linewidth of either system [32-34]. This can be verified via Ramsey interferometry as in Fig. 1(b), examining a Fourier transform of the Ramsey oscillations to reveal the magnon-number splitting of the qubit spectrum as in Fig. 1(c) [16,21,39]. The shift per excitation is $2 \chi_{q-m} / 2 \pi=-3.48 \mathrm{MHz}$, compared to the qubit linewidth $\gamma_{q} / 2 \pi=0.36 \mathrm{MHz}$ and magnon linewidth $\gamma_{m} / 2 \pi=1.6 \mathrm{MHz}$. Magnons in the Kittel mode induce both increased dephasing and a continuous frequency shift of the qubit. However, for the qubit resonance for the magnon Fock state $n_{m}=0$, only the former remains in the strong dispersive regime as the latter is suppressed $[32,39]$.

Sensing of a steady-state population of a coherent state of magnons in the Kittel mode is carried out by performing Ramsey interferometry on the qubit. The sensitivity $\mathcal{S}$ is defined as the smallest measurable value of the magnon population that can be detected with a unit signal-to-noise ratio over a one-second integration time [1]. Figure 1(d) shows the magnon detection sensitivity $\mathcal{S}$ calculated from an analytical model as a function of the magnon linewidth $\gamma_{m}$ for two values of the dispersive shift amplitude $\left|\chi_{q-m}\right|$, with all other parameters similar to those in the experiment [39]. For values up to $\gamma_{m} \approx 4\left|\chi_{q-m}\right|$, increasing the magnon linewidth improves the sensitivity with a scaling $\mathcal{S} \sim 1 / \gamma_{m}$, demonstrating that the sensing is governed primarily by dissipation in the Kittel mode. Notably, within the strong dispersive regime with $\gamma_{m}<2\left|\chi_{q-m}\right|$, further increasing the amplitude of the dispersive shift $\left|\chi_{q-m}\right|$ has a negligible effect on the sensitivity as the magnon-number peaks are already sufficiently resolved.

The sensitivity is benchmarked by using a microwave drive applied near resonance with the Kittel mode to excite a coherent state of magnons with an average population $\bar{n}_{m}$ with the qubit in the ground state [Fig. 1(b)]. The magnon population excited by a given drive amplitude is calibrated from the qubit spectrum as in Fig. 1(c) [39]. During the continuous magnon excitation, two $\pi / 2$ pulses around the same axis are applied to the qubit, separated by a free evolution time corresponding to the sensing time $\tau$. At the end of the sequence, the qubit state is measured using the high-power readout technique [45]. Measurements of the qubit state are taken over $10^{4}$ to $10^{6}$ averages. For $\bar{n}_{m}=0$, the probability of the qubit being in the excited state $p_{e}\left(\bar{n}_{m}=0\right)$ is determined primarily by the sensing 
(a)

(b)
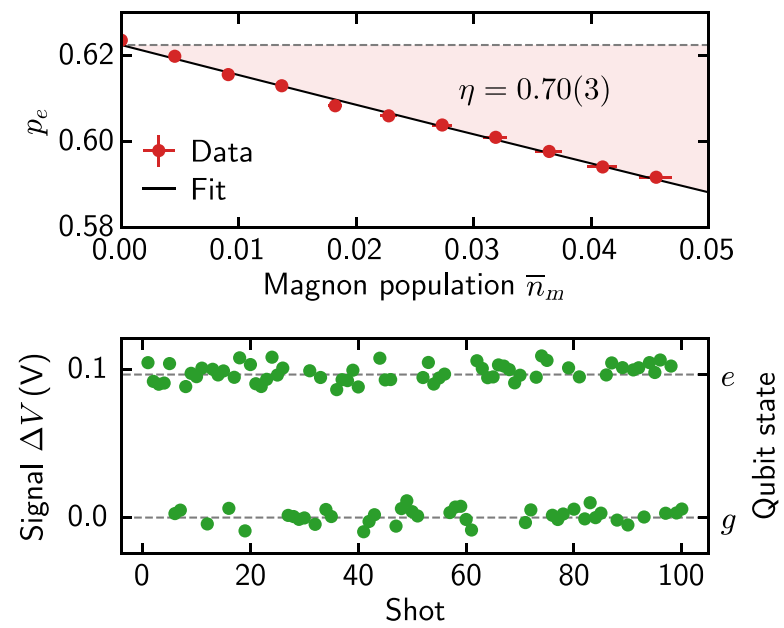

(c)

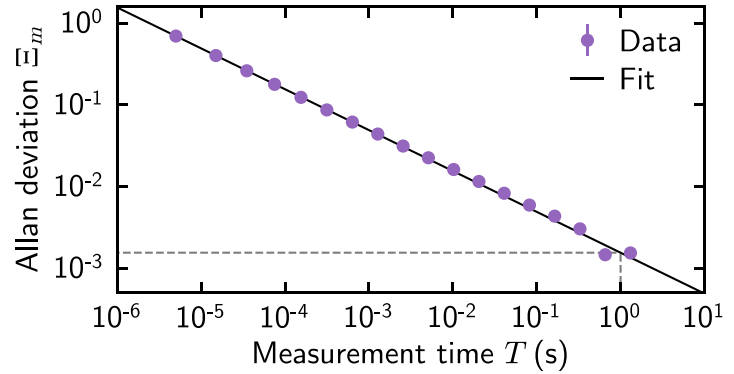

FIG. 2. (a) Qubit excited-state probability $p_{e}$ as a function of the magnon population $\bar{n}_{m}$. Equation (1) is fitted to the data to yield the magnon detection efficiency $\eta=0.70$. (b) Demodulated signal $\Delta V$ for a subset of single-shot qubit measurements. Horizontal dashed lines indicate the demodulated signal corresponding to the qubit in the ground state $|g\rangle$ and excited state $|e\rangle$. (c) Magnon population Allan deviation $\Xi_{m}$ as a function of measurement time $T$. The solid line indicates a fit to Eq. (2). The horizontal dashed line indicates the value of $\Xi_{m}$ for $T=1 \mathrm{~s}$ (vertical dashed line), corresponding to the magnon detection sensitivity $\mathcal{S}=1.55 \times 10^{-3}$ magnons $/ \sqrt{\mathrm{Hz}}$.

time $\tau$, qubit drive detuning $\Delta_{s}$, and the initialization, control, and readout errors. Due to the dispersive interaction, $p_{e}\left(\bar{n}_{m}>0\right)$ depends on the magnon population $\bar{n}_{m}$, as in Fig. 2(a) for $\tau=0.8 \mu$ s and qubit drive detuning $\Delta_{s}=0$. For $\bar{n}_{m} \ll 1$, this dependence can be approximated with

$$
p_{e}\left(\bar{n}_{m}\right)=p_{e}(0) \pm \eta \bar{n}_{m},
$$

where the sign depends on the qubit drive detuning and sensing time, and $\eta$ is the efficiency of detecting magnons with the qubit, defined to be positive [39]. A fitted value of $\eta=0.70(3)$ is obtained from the data of Fig. 2(a). The sensing signal corresponds to the difference between the probability $p_{e}$ with and without magnons present in the Kittel mode. As the qubit excitation probability is bounded and converges to a fixed value, values of $\bar{n}_{m}$ sufficiently larger than those used here lead to detector saturation and a loss of validity of the linear model of Eq. (1). The saturation point can be adjusted by modifying the dispersive shift in situ, at the expense of the sensitivity. Here, the device is configured to achieve the best possible sensitivity.

To characterize the noise associated with the sensing procedure, repeated single-shot readout of the qubit is carried out, shown in Fig. 2(b). Such a measurement also allows for quantifying the fidelity of the qubit readout, here estimated at around $90 \%$ [39]. The scaling of sensor noise for different measurement times is given by the Allan deviation as shown in Fig. 2(c), which can be expressed in terms of the qubit probability, $\Xi_{q}(T)$, or the magnon population, $\Xi_{m}(T)=\Xi_{q}(T) / \eta$. This corresponds to the standard deviation of subsets of the data as a function of measurement time $T=N \tau_{\text {total }}$, where $N$ is the number of shots and $\tau_{\text {total }}=5 \mu \mathrm{s}$ is the duration of a single sequence. As shown in Fig. 2(c), the sensitivity $\mathcal{S}$ is related, by definition, to the Allan deviation via

$$
\Xi_{m}(T)=\mathcal{S} / \sqrt{T} .
$$

Fitting Eq. (2) to the data yields a sensitivity of $\mathcal{S}=1.55(5) \times 10^{-3}$ magnons $/ \sqrt{\mathrm{Hz}}$, which corresponds to a microwave magnetic field sensitivity of $1.86 \times$ $10^{-15} \mathrm{~T} / \sqrt{\mathrm{Hz}}$ [39]. The data is seen not to deviate significantly from the scaling up to $T>1 \mathrm{~s}$, implying that the noise floor due to slow fluctuations has not been reached and scaling the noise to $T=1 \mathrm{~s}$ is valid. Numerical simulations modeling the dispersive qubit-magnon interaction and imitating the experimental protocol yield a sensitivity of $1.35 \times 10^{-3}$ magnons $/ \sqrt{\mathrm{Hz}}$, showing excellent agreement with the experimental results. A single fitting parameter is used, but it is seen not to affect the value of the sensitivity significantly at zero detuning [39]. The underestimation of the sensitivity by the simulation is probably due to additional interactions that are not accounted for in the dispersive Hamiltonian considered in the simulations.

The sensing time $\tau$ is a fundamental parameter that can be used to investigate the performance of the sensor. The efficiency $\eta$ is measured as a function of sensing time $\tau$, as in Fig. 3(a) for $\Delta_{s}=0$. This reveals a weak optimum, as information acquisition saturates rapidly but qubit decoherence has a relatively slower falloff with $T_{2}^{*}=0.89 \mu \mathrm{s}$. Numerical simulations, the results of which are shown as a solid line in Fig. 3(a), are in good agreement with the data and reproduce all essential features. The oscillations are due to dynamical detuning of the magnon frequency as the excited state of the qubit is populated during the sensing sequence.

Characterization of the noise associated with repeated measurements is also carried out as a function of sensing time, calculating the Allan deviation of the qubit excitation probability for a one-second measurement time, $\Xi_{q}(T=1 \mathrm{~s})$, as shown in Fig. 3(b) for $\Delta_{s}=0$. The solid line in Fig. 3(b) shows the qubit shot noise [1] given by 

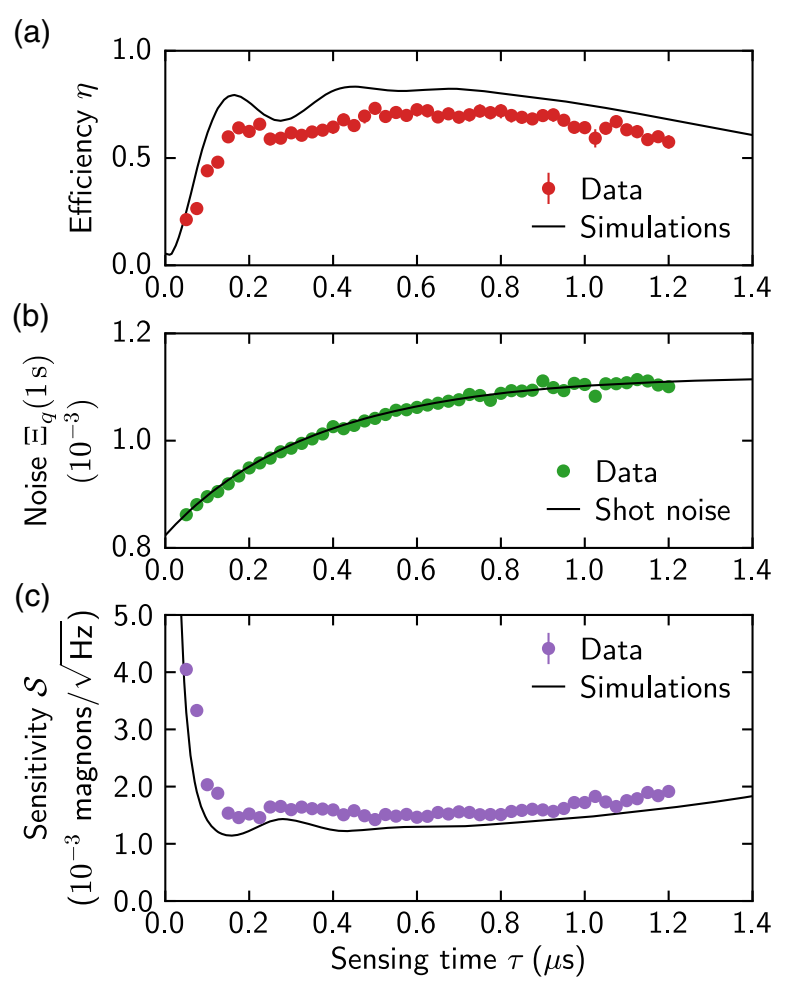

FIG. 3. (a) Magnon detection efficiency $\eta$, (b) noise of singleshot qubit readout for one-second measurement time, $\Xi_{q}(1 \mathrm{~s})$, and (c) magnon detection sensitivity $\mathcal{S}$ as a function of sensing time $\tau$. In (a) and (c), solid black lines are results from numerical simulations. In (b), the solid black line is the shot noise calculated from Eq. (3).

$$
\Xi_{q}(T=1 \mathrm{~s})=\sqrt{p_{e}\left(1-p_{e}\right) \frac{\tau_{\text {total }}}{1 \mathrm{~s}}}
$$

where $p_{e}=p_{e}\left(\bar{n}_{m}=0\right)$. This shows excellent agreement between theory and experiment, as the high-fidelity projective qubit readout is shot-noise limited as noise from components such as amplifiers has been digitized, ensuring that the qubit readout process is not the dominant constraint on the magnon detection sensitivity. Figure 3(c) shows the sensitivity $\mathcal{S}$ as a function of the sensing time $\tau$ obtained from the efficiency $\eta$ and noise $\Xi_{q}$. The optimal sensing time is seen to be approximately half of the qubit coherence time $T_{2}^{*}=0.89 \mu \mathrm{s}$, with the best sensitivity $\mathcal{S}=1.42(3) \times$ $10^{-3}$ magnons $/ \sqrt{\mathrm{Hz}}$ measured for $\tau=0.5 \mu \mathrm{s}$. Such an optimal time is equivalent to that of procedures based on sensing via a frequency shift [1].

The relative contributions of dephasing and frequency shifts to the sensitivity are revealed in this case by examining the Ramsey fringes as in Fig. 4(a). Here, these are measured by sweeping the relative detuning $\Delta_{s}$ between the frequencies of the qubit and the $\pi / 2$ pulses applied to the qubit during the sensing protocol. The Ramsey fringes show a reduction of contrast when a finite magnon population is present in the Kittel mode, but do not exhibit
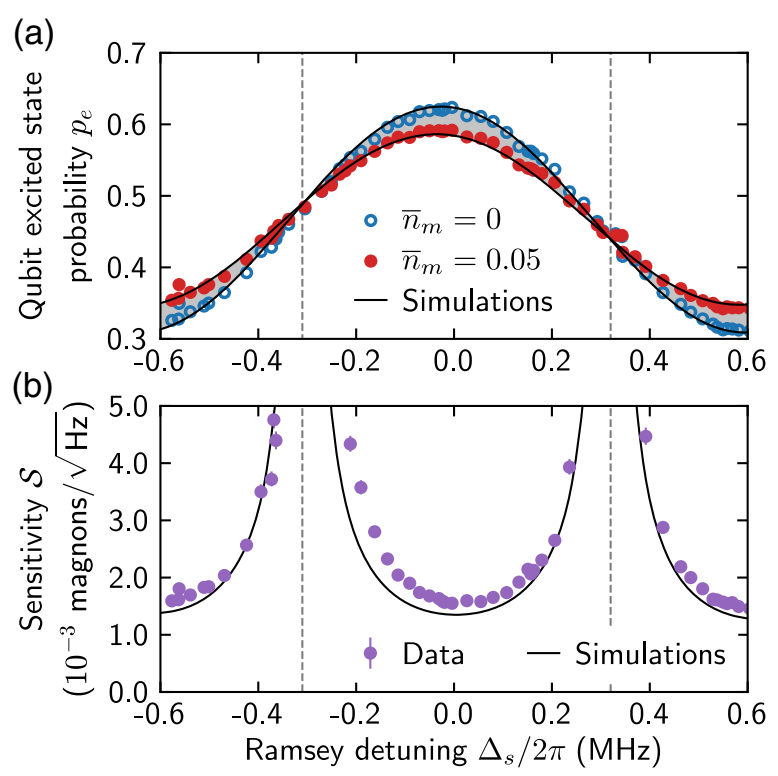

FIG. 4. (a) Qubit excited-state probability $p_{e}$ and (b) magnon detection sensitivity $\mathcal{S}$ as a function of Ramsey detuning $\Delta_{S} / 2 \pi$. The probability $p_{e}$ is shown for the cases of sensing different steady-state magnon populations $\bar{n}_{m}=0$ (blue circles) and $\bar{n}_{m}=$ 0.05 (red dots), with the shaded region indicating the difference. Dashed vertical lines correspond to nodes of the Ramsey fringes. Solid black lines are results from numerical simulations.

a significant frequency shift. Figure 4(b) shows that the optimal sensitivity is obtained near zero detuning, and becomes asymptotically worse when approaching detunings corresponding to nodes of the Ramsey fringes. Consequently, the dissipation of magnons is verified as the dominant mechanism by which the qubit is sensitive to the magnon population within the strong dispersive regime, and increasing the linewidth of the Kittel mode up to $\gamma_{m} \approx$ $4\left|\chi_{q-m}\right|$ would improve the sensitivity by as much as a factor of three without other changes [39].

These conclusions reflect the qualitative behavior explored in Fig. 1(d), and thus the procedure presented here falls under the category of sensing techniques known as $T_{2}^{*}$ relaxometry [1]. Such a conclusion contrasts with previous approaches such as in Ref. [16], where the sensing of magnons is based on entanglement of the qubit and Kittel mode and is thus greatly hindered by an increased magnon dissipation rate, even in the strong dispersive regime.

The demonstrated level of sensitivity represents a significant advancement relative to existing magnon detection schemes, where the typical quantity of detected magnons is many orders of magnitude larger $[28,29,46]$. Furthermore, detection on the level of single magnons and below can be useful for probing magneto-optical effects in the quantum regime as part of the development of quantum transducers $[21,23]$, and may be used in dark matter searches for axionlike particles [47-50]. The device can also be used as a static magnetic field sensor, as the detuning of the Kittel 
mode by such a field is measurable by monitoring the magnon population excited by a fixed microwave drive. In this way, the magnetic-field-insensitive transmon qubit can be made sensitive to the applied field in a controlled manner. Interactions beyond the dispersive qubit-magnon interaction, such as that due to the cavity-magnon crossKerr interaction [21,51], could also be utilized for magnon sensing. They could potentially offer further improvements in sensitivity alongside enabling continuous sensing.

In conclusion, we have demonstrated that probing the coherence of a superconducting transmon qubit dispersively coupled to a magnetostatic mode allows for quantum sensing of magnons with a sensitivity on the order of $10^{-3}$ magnons $/ \sqrt{\mathrm{Hz}}$. The device parameters and operating point of the protocol lead to the qubit becoming sensitive to the magnon population primarily through magnon decay. Counterintuitively, this results in the detection sensitivity being improved by increasing losses in the magnetostatic mode while operating close to or in the strong dispersive regime. The results presented here constitute an advancement in the detection and characterization of small magnon populations, and are also applicable to other physical systems in microwave quantum optics [6-8] and quantum acoustics [13-15], for example. The protocol demonstrated here therefore provides tools for a broad range of fields, from magnon spintronics to quantum sensing and hybrid quantum systems.

The authors would like to thank Arjan van Loo for valuable discussions, as well as the photograph of the YIG sphere. This work was partly supported by JSPS KAKENHI (Grant No. 18F18015), JST ERATO (Grant No. JPMJER1601), and FRQNT Postdoctoral Fellowships. S. P. W. was supported by the MEXT Monbukagakusho Scholarship. D. L.-Q. received support as an International Research Fellow of JSPS.

*swolski@qc.rcast.u-tokyo.ac.jp

†yasunobu@ap.t.u-tokyo.ac.jp

${ }^{*}$ Present address: Nord Quantique, Sherbrooke, Québec J1K 0A5, Canada

[1] C. L. Degen, F. Reinhard, and P. Cappellaro, Rev. Mod. Phys. 89, 035002 (2017).

[2] J. Bylander, S. Gustavsson, F. Yan, F. Yoshihara, K. Harrabi, G. Fitch, D. G. Cory, Y. Nakamura, J.-S. Tsai, and W. D. Oliver, Nat. Phys. 7, 565 (2011).

[3] A. Narla, S. Shankar, M. Hatridge, Z. Leghtas, K. M. Sliwa, E. Zalys-Geller, S. O. Mundhada, W. Pfaff, L. Frunzio, R. J. Schoelkopf, and M. H. Devoret, Phys. Rev. X 6, 031036 (2016).

[4] K. Inomata, Z. Lin, K. Koshino, W. D. Oliver, J.-S. Tsai, T. Yamamoto, and Y. Nakamura, Nat. Commun. 7, 12303 (2016).

[5] S. Kono, K. Koshino, Y. Tabuchi, A. Noguchi, and Y. Nakamura, Nat. Phys. 14, 546 (2018).

[6] M. Kristen et al., npj Quantum Inf. 6, 57 (2020).
[7] Z. Wang et al., arXiv:1909.12295.

[8] T. Hönigl-Decrinis, R. Shaikhaidarov, S. E. de Graaf, V. N. Antonov, and O. V. Astafiev, Phys. Rev. Applied 13, 024066 (2020).

[9] A. Blais, R.-S. Huang, A. Wallraff, S. M. Girvin, and R. J. Schoelkopf, Phys. Rev. A 69, 062320 (2004).

[10] A. Wallraff, D. I. Schuster, A. Blais, L. Frunzio, R.-S. Huang, J. Majer, S. Kumar, S. M. Girvin, and R. J. Schoelkopf, Nature (London) 431, 162 (2004).

[11] G. Kurizki, P. Bertet, Y. Kubo, K. Mølmer, D. Petrosyan, P. Rabl, and J. Schmiedmayer, Proc. Natl. Acad. Sci. U.S.A. 112, 3866 (2015).

[12] A. A. Clerk, K. W. Lehnert, P. Bertet, J. R. Petta, and Y. Nakamura, Nat. Phys. 16, 257 (2020).

[13] K. J. Satzinger et al., Nature (London) 563, 661 (2018).

[14] Y. Chu, P. Kharel, T. Yoon, L. Frunzio, P. T. Rakich, and R. J. Schoelkopf, Nature (London) 563, 666 (2018).

[15] P. Arrangoiz-Arriola, E. A. Wollack, Z. Wang, M. Pechal, W. Jiang, T. P. McKenna, J. D. Witmer, R. Van Laer, and A. H. Safavi-Naeini, Nature (London) 571, 537 (2019).

[16] D. Lachance-Quirion, S. P. Wolski, Y. Tabuchi, S. Kono, K. Usami, and Y. Nakamura, Science 367, 425 (2020).

[17] A. G. Gurevich and G. A. Melkov, Magnetization Oscillations and Waves (CRC press, Boca Raton, 1996).

[18] D. D. Stancil and A. Prabhakar, Spin Waves (Springer, Boston, 2009).

[19] Y. Tabuchi, S. Ishino, A. Noguchi, T. Ishikawa, R. Yamazaki, K. Usami, and Y. Nakamura, Science 349, 405 (2015).

[20] Y. Tabuchi, S. Ishino, A. Noguchi, T. Ishikawa, R. Yamazaki, K. Usami, and Y. Nakamura, C.R. Phys. 17, 729 (2016).

[21] D. Lachance-Quirion, Y. Tabuchi, A. Gloppe, K. Usami, and Y. Nakamura, Appl. Phys. Express 12, 070101 (2019).

[22] J. A. Haigh, A. Nunnenkamp, A. J. Ramsay, and A. J. Ferguson, Phys. Rev. Lett. 117, 133602 (2016).

[23] R. Hisatomi, A. Osada, Y. Tabuchi, T. Ishikawa, A. Noguchi, R. Yamazaki, K. Usami, and Y. Nakamura, Phys. Rev. B 93, 174427 (2016).

[24] X. Zhang, C. L. Zou, L. Jiang, and H. X. Tang, Sci. Adv. 2, e1501286 (2016).

[25] S. Viola Kusminskiy, H. X. Tang, and F. Marquardt, Phys. Rev. A 94, 033821 (2016).

[26] Y.-P. Wang, J. W. Rao, Y. Yang, P.-C. Xu, Y. S. Gui, B. M. Yao, J. Q. You, and C.-M. Hu, Phys. Rev. Lett. 123, 127202 (2019).

[27] E. Saitoh, M. Ueda, H. Miyajima, and G. Tatara, Appl. Phys. Lett. 88, 182509 (2006).

[28] Y. Kajiwara et al., Nature (London) 464, 262 (2010).

[29] A. V. Chumak, A. A. Serga, M. B. Jungfleisch, R. Neb, D. A. Bozhko, V. S. Tiberkevich, and B. Hillebrands, Appl. Phys. Lett. 100, 082405 (2012).

[30] S. O. Demokritov, B. Hillebrands, and A. N. Slavin, Phys. Rep. 348, 441 (2001).

[31] T. Sebastian, K. Schultheiss, B. Obry, B. Hillebrands, and H. Schultheiss, Front. Phys. 3, 35 (2015).

[32] J. Gambetta, A. Blais, D. I. Schuster, A. Wallraff, L. Frunzio, J. Majer, M. H. Devoret, S. M. Girvin, and R. J. Schoelkopf, Phys. Rev. A 74, 042318 (2006).

[33] D. I. Schuster et al., Nature (London) 445, 515 (2007). 
[34] D. Lachance-Quirion, Y. Tabuchi, S. Ishino, A. Noguchi, T. Ishikawa, R. Yamazaki, and Y. Nakamura, Sci. Adv. 3, e1603150 (2017).

[35] Y. Tabuchi, S. Ishino, T. Ishikawa, R. Yamazaki, K. Usami, and Y. Nakamura, Phys. Rev. Lett. 113, 083603 (2014).

[36] H. Huebl, C. W. Zollitsch, J. Lotze, F. Hocke, M. Greifenstein, A. Marx, R. Gross, and S. T. B. Goennenwein, Phys. Rev. Lett. 111, 127003 (2013).

[37] J. Koch, T. M. Yu, J. Gambetta, A. A. Houck, D. I. Schuster, J. Majer, A. Blais, M. H. Devoret, S. M. Girvin, and R. J. Schoelkopf, Phys. Rev. A 76, 042319 (2007).

[38] H. Paik et al., Phys. Rev. Lett. 107, 240501 (2011).

[39] See the Supplemental Material at http://link.aps.org/ supplemental/10.1103/PhysRevLett.125.117701 for additional details of the experimental apparatus and methodology, data analysis, and numerical simulations, which includes Refs. [40-44].

[40] N. F. Ramsey, Phys. Rev. 78, 695 (1950).
[41] J. R. Johansson, P. D. Nation, and F. Nori, Comput. Phys. Commun. 184, 1234 (2013).

[42] F. Motzoi, J. M. Gambetta, P. Rebentrost, and F. K. Wilhelm, Phys. Rev. Lett. 103, 110501 (2009).

[43] http://labber.org.

[44] https://github.com/qipe-nlab/Labber-PSICT.

[45] M. D. Reed, L. DiCarlo, B. R. Johnson, L. Sun, D. I. Schuster, L. Frunzio, and R. J. Schoelkopf, Phys. Rev. Lett. 105, 173601 (2010).

[46] J. Cramer et al., Nat. Commun. 9, 1089 (2018).

[47] R. Barbieri, C. Braggio, G. Carugno, C. S. Gallo, A. Lombardi, A. Ortolan, R. Pengo, G. Ruoso, and C. C. Speake, Phys. Dark Universe 15, 135 (2017).

[48] N. Crescini et al., Eur. Phys. J. C 78, 703 (2018).

[49] G. Flower, J. Bourhill, M. Goryachev, and M. E. Tobar, Phys. Dark Universe 25, 100306 (2019).

[50] N. Crescini et al., Phys. Rev. Lett. 124, 171801 (2020).

[51] F. Helmer, M. Mariantoni, E. Solano, and F. Marquardt, Phys. Rev. A 79, 052115 (2009). 